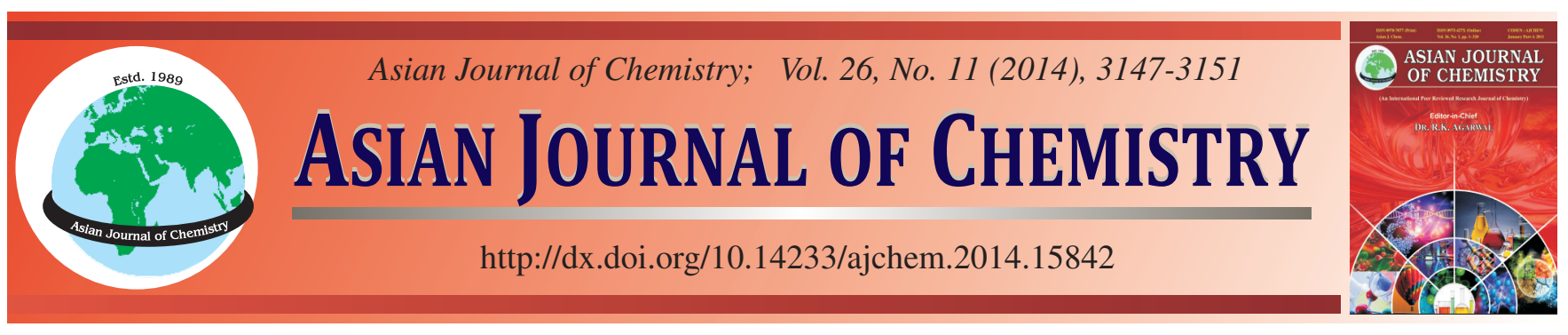

\title{
Purification and Establishment of HPLC Method for Numb-Taste Components from Sichuan Huajiao (Zanthoxylum bungeanum)
}

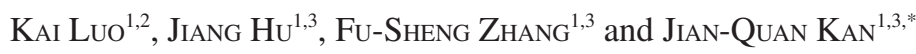

${ }^{1}$ College of Food Science, Southwest University, Chongqing 400715, P.R. China

${ }^{2}$ Chongqing Three Gorges University, Chongqing 404000, P.R. China

${ }^{3}$ Laboratory of Ministry of Agriculture for Quality and Safety Risk Assessment of Produce Storage and Preservation, Chongqing 400715, P.R. China

*Corresponding author: Tel/Fax: +86 23 68250375; E-mail: kanjianquan@163.com

Through a new and simple chromatographic technique combination of preparative liquid chromatography, the numb-taste component of huajiao was isolated. At the same time, used GC-MS method to qualitative detection of the separated material, this substance was determined just as numb-taste components. Using this material as a standard reference material, established a HPLC detection method of numb-taste components of Huajiao. Through experimental analysis found that this method has better precision. The limit of detection was $0.044 \mu \mathrm{g} / \mathrm{mL}$, the recovery rate was in the range 88.1-104.9\% and relative standard deviation was in the range 0.79-2.39\%. This establishment of detection method provided excellent technical for identifying the quality of Huajiao.

Keywords: Sichuan Huajiao, HPLC, Numb-taste Component.

\section{INTRODUCTION}

Sichuan Huajiao, the pericarps of the fruits of Zanthoxylum bungearnum Maxim. Huajiao is a large and complex genus that includes about 250 species in mainly tropical and temperate regions of the world. which is native to southwest China in Sichuan, Yunnan, Guizhou, Gansu, Shanxi, Tibet, Guangxi, Guangdong and Chongqing. This utilized as a pungent food stuff and also listed in Ben Cao Gang Mu and Ben Cao Jing as a kind of traditional Chinese medicine for treatment of vomiting, tooth ache, stomach ache due to cold in the stomach and abdominal pain due to roundworm and so on ${ }^{1}$. The fruits and leaves of Zanthoxylum bungeanum have reported to contain aliphatic amides ${ }^{2-8}$, alkaloids, lignans, flavonoids ${ }^{9-11}$, alkyl amide and terpenoids ${ }^{12,13}$. The materials composition of Huajiao peel is very complex. It also includes essential oil, alkaloid, amide, lingams, coumarone, falconoid and fatty acid, $e t c^{14}$. In the past, Chinese Huajiao is mainly used as a pungent food stuff, however, as a kind of additive, we don't know how to evaluate the quality of them. Because there is no pure of numb-taste components of Huajiao and also there is no standard testing method and index about Huajiao. Therefore, selection of Huajiao mainly by virtue of the senses and experience, this method is not precise and no uniform standard. In this study, through certain means of separation, we have got the standard control sample of numb-taste component in
Huajiao. Using this standard control sample, we have established a HPLC detection method for the detection of numb-taste component of Huajiao. These studies will be essential for Huajiao quality detection to provide effective technical support. According to the research results, we basically to build numbtaste components of Huajiao testing standards.

\section{EXPERIMENTAL}

The pericarps of $Z$. bungeanum Maxim. (samples 1-8) used in this work were purchased from a local market in Chongqing Province, China. Sample 1 was used in all other experiments. Pericarps of $Z$. bungeanum Maxim. were crushed with a disintegrator (FW-100 Test Instrument Co., Ltd, Tianjin, China) and then filtered through 40 mesh sieve. The sample powders collected were stored in the desiccator before analysis. The HPLC instrument used was a Shimadzu LC-3A high performance liquid chromatograph, equipped with a SPD-1 multi-wavelength UV detector (Shimadzu), a UVD-2 UV detector $(\lambda=254 \mathrm{~nm})$ and an RF-510 fluorescence detector (Shimadzu). The instrument used to determine UV absorbance was a Shimadzu UV-310 ultraviolet spectrometer. Methanol as the mobile phase was an analytical grade reagent produced by Tianjin Chemical Reagent Factory (China). The experiment used silica gel were purchased in the sea company (Qingdao Shandong Province, China). Water was triply distilled. The extraction and concentration system was assembled in our 
laboratory and the schematic diagram is shown in Fig. 1. Electric heating sleeves were made in our laboratory.

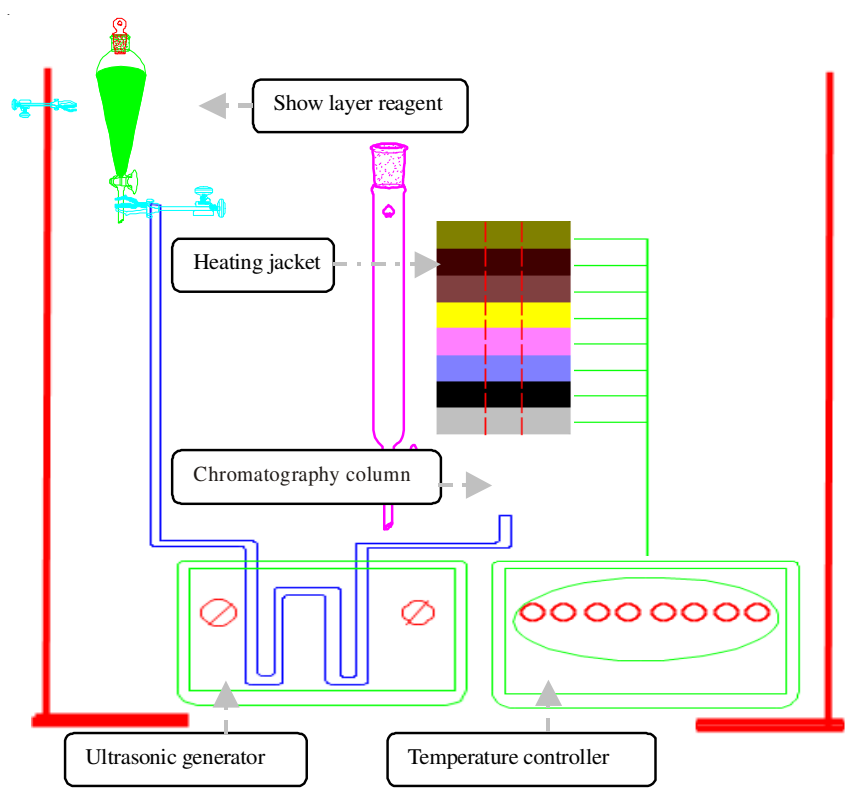

Fig. 1. Improved dry column chromatography system

\section{Extraction and isolation}

Preparation of the standard samples for numb-taste components of Huajiao: The first step: preparation oil resin of Huajiao. The pericarps of Huajiao were crushed with a disintegrator and then filtered through 40 mesh sieve. supercritical carbon dioxide extraction method was used to extract of Huajiao oil resin. Extraction conditions were that the raw material granularity was 40 , extraction time was $3 \mathrm{~h}$, flow rate was $15 \mathrm{~kg} / \mathrm{h}$, temperature was $45^{\circ} \mathrm{C}$, pressure was $30 \mathrm{MPa}$. The second step: countercurrent dry column chromatography. The Huajiao oil resin and silica gel (two times of oil resin) were mixed, dried as raw material. Selection of chromatography column $(500 \times 26 \mathrm{~mm}$, with a converting joint and a sand core baffle). In packing column, in order to ensure that the silica gel filling evenly and closely, we must to gently tap the column. When packed column height reached $1 \mathrm{~cm}$ began to fill materials, the materials also packed $1 \mathrm{~cm}$ height. Subsequently we joined amount of silica gel to make the column height achieve about $40 \mathrm{~cm}$. Finally, the column and the dropping funnel will be connected through the catheter. At the same time, developing agent (6\% acetone-chloroform solution) were loaded into the drop funnel. Traditional methods can only make separation height achieve $24 \mathrm{~cm}$, so a number of material is difficult to be separated, especially about a lot of structurally similar material. If pressure was increased, the silica gel in column will be promoted. So, in order to make those structurally similar materials to be separated thoroughly, we designed a new device. This device was shown in Fig. 1. When the developing agent flows down from the dropping funnel, it will be preheated and ultrasonic treatment in the catheter. With Huajiao raw material extended layer by layer, due to rising resistance increase gradually, all sorts of material transfer rate will gradually decrease, so, we designed a new heating method to make the column maintain different temperature. It can speed up the separation of substances. Moreover, the material can be separated more thoroughly. The third step: After each layer exhibited, it will be separated by the various ribbon. The different color silica gel were placed in the fume hood so that the solvent would be evaporated over, this can get to the 8 portion of the components (Ds1-Ds8). Detection of each part in $254 \mathrm{~nm}$ absorption value and collection the part of the largest light absorption value as a crude product (Ds-A).

Preparation of high-performance liquid chromatography (pre-HPLC) purified coarse crystal. The coarse crystalline (DsA $0.2 \mathrm{~g}$ ) dissolve in $5 \mathrm{~mL}$ methanol, through the pre-HPLC to purification. Preparation conditions were that, the column was Shim-PACK pre-ODS $(250 \times 20 \mathrm{~mm})$, the eluting agent was $70 \%$ methanol-water solution, flow velocity $(5 \mathrm{~mL} / \mathrm{min})$, the column temperature (room temperature), the UV detection wavelength was $254 \mathrm{~nm}$, automatic sampling and each sample was $0.1 \mathrm{~mL}$. The eluent of maximum absorption peak will be collected by artificial. Use the rotary evaporator to out of methanol and with the right amount of heat petroleum ether to dissolve them. Put them in the refrigerator freezing to crystallization $\left(-18{ }^{\circ} \mathrm{C}\right)$, the numb- taste component crystals can be get in this way (PC2).

GC-MS determination and appraisal of crystalline purity (PC2): The purity of the crystalline (PC2) can be determined by GC-MS. GC analysis was accomplished on a HP5890-5989A gas chromatograph using a silica capillary column $(0.25 \mathrm{~m} \times 30 \mathrm{~m} \times 0.32 \mathrm{~mm}, 100 \%$ polyethylene glycol used as stationary phase). Nitrogen was used as carrier gas $(35.0 \mathrm{~cm} / \mathrm{s})$ with an equilibrium time of $3 \mathrm{~min}$ before each run. Basically, the injector temperature was $250{ }^{\circ} \mathrm{C}$, The column temperature was initially kept at $70{ }^{\circ} \mathrm{C}$ for $5 \mathrm{~min}$ and then increased to $280{ }^{\circ} \mathrm{C}$ at $5{ }^{\circ} \mathrm{C} / \mathrm{min}$ and held for $5 \mathrm{~min}$. Crystal purity is determined by the ratio of objective the peak area and all of the response peak area (except solvent peak area). The MS scan range was $m / z$ 35-550 with a scan interval of 0.5 $\mathrm{s}$, a scan speed of $1000 \mathrm{u} / \mathrm{s}$ and a detector voltage of $0.9 \mathrm{kV}$. The ion source temperature was $200{ }^{\circ} \mathrm{C}$ with an interface temperature of $250{ }^{\circ} \mathrm{C}$. The solvent cut time was $6 \mathrm{~min}$.

Establishment a quantitative method for numb-taste component of HPLC (external standard method): Use the purified crystal (PC2) as the standard substance to establish the method of HPLC. The column used was a Shim-PACK ODS, $250 \times 4 \mathrm{~mm}, 3 \mu \mathrm{m}$ at $45^{\circ} \mathrm{C}$. The flow rate was $0.80 \mathrm{~mL} /$ min and the injection volume used was $15 \mu \mathrm{L}$. The UV detection wavelength was $254 \mathrm{~nm}$. Water and methanol were used as the mobile phase. Using the gradient elution method, in the first $15 \mathrm{~min}$, water and methanol were $1: 1$, from sixteenth min to $25 \mathrm{~min}$, water and methanol were $3: 7$, at the last minute, water and methanol were $1: 1$. Technical index about detection limit, accuracy degree and precision were studied respectively.

\section{RESULTS AND DISCUSSION}

Separation and purification of numb-taste components of Huajiao: Countercurrent dry column chromatography is an improved chromatographic technique. Using this method can quickly obtain preparative separation and the effect with the same of thin layer chromatography. At first, the traditional method of column chromatographic was used to separate the 
numb-taste components. However, it was found that the column separation height can reach only $23 \mathrm{~cm}$. At that height, various substances can not be separated perfect, especially for the structural similarity of numb-taste components, a number of material will overlap. In order to make all of numb-taste components separated perfect, the new method was used for separation (Fig. 1). Based on the developing agents to ultrasound and heat treatment, can make the developing agent have better performance for development layer. In order to make the exhibition layer height get increased under no increase the pressure condition, at the same time, exhibition layer velocity get accelerated, the heating device was used to make the column maintain different temperature at different stages. Using this device, column chromatography silica gel can be clearly divided into 8 sections (Ds1-Ds8), no mutual interference around them. Through the determination of the light absorption value in $254 \mathrm{~nm}$, found Ds3 has a maximum absorption value (Fig. 2), which was used as a crude product (Ds-A). For developing agent, with a large number of tests, it was found that $6 \%$ acetone-chloroform solution was better than the others.

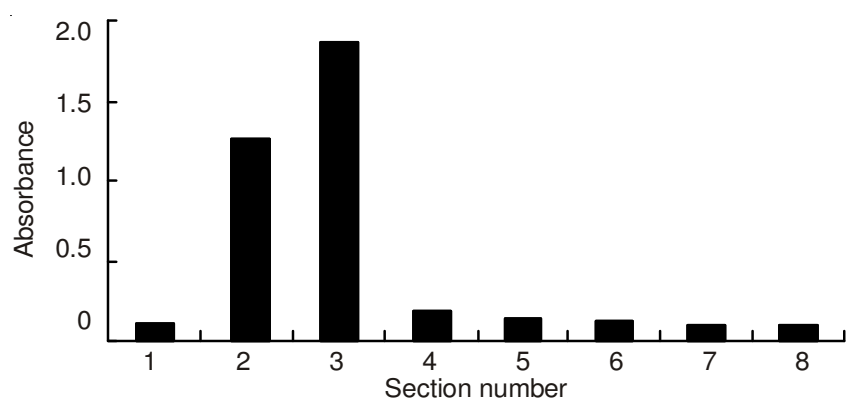

Fig. 2. Absorbance of every section after dry-column chromatography

In order to further purification of numb-taste components, pre-HPLC technique was adopted. It was found that using $50 \%$ methanol-water solvent elution, 70 min was still unable to elute out of numb-taste components through previous research found, using $60 \%$ and $70 \%$ all can elute out of it, the time was $1 \mathrm{~h}$ and $0.5 \mathrm{~h}$ respectively. Elution peak shape was just as shown in Fig. 3. If using $80 \%$ methanol-water solvent, the elution peak shape was not smooth. After comprehensive consideration, $70 \%$ methanol-water solvent was used as mobile phase. It was found that the solution of elution peak in the 206

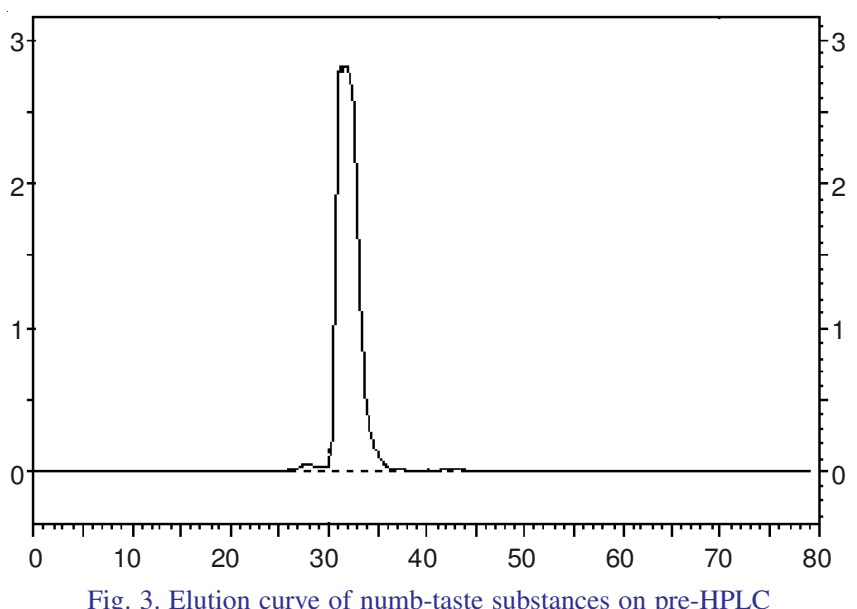

Fig. 3. Elution curve of numb-taste substances on pre-HPLC and 254 had obvious absorption peak by ultraviolet scanning analysis, which consistent with the literature about unsaturated amides. The numb-taste component crystals can be get in this way (PC2).

GC-MS determination and appraisal of crystalline purity (PC2): In order to further clarify the nature of the PC2, it was carried out by gas chromatography mass spectrometry (Fig. 4). These results were consistent with the literatures. These substances were unsaturated amides of Huajiao. At the same time, which purity could reach $95 \%$.

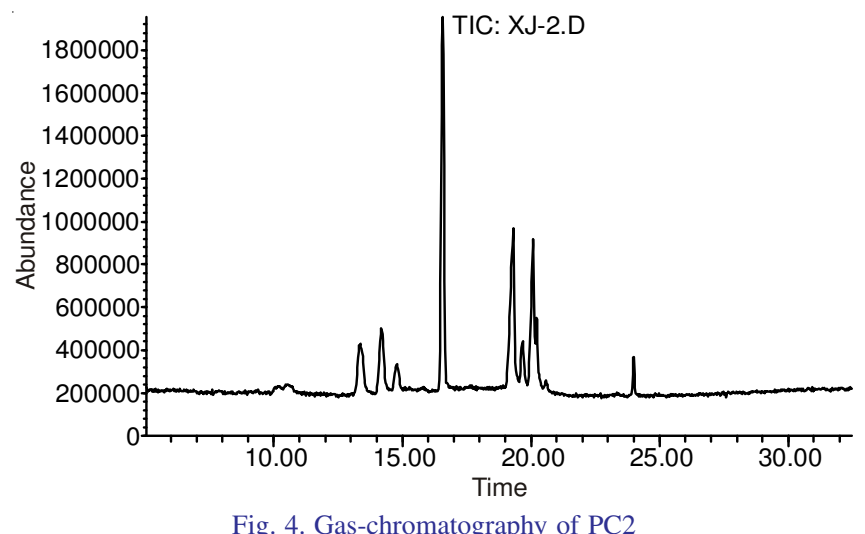

Establishment of a quantitative method for numb-taste component of HPLC: The theory of using HPLC method to detection numb-taste components content is based on the amount of substance through the detector was proportional to detector response signal, using peak area size can effectively solute content in reaction solution. Previous research has discovered that water and methanol were used as the mobile phase and using the gradient elution method, in the first 15 min, water and methanol were $1: 1$, from $16 \mathrm{~min}$ to $25 \mathrm{~min}$, water and methanol were 3:7, at the last minute, water and methanol were $1: 1$. The effect of column temperature on the separation was analyzed. In the column temperature at $25^{\circ} \mathrm{C}$ (room temperature), 30, 35, 40, and $45^{\circ} \mathrm{C}$ were studied and found that the column temperature at $40{ }^{\circ} \mathrm{C}$, elution peak time interval was maximum and theoretical plate number was maximum.

Draw of HPLC standard curve: Methanol was used for the preparation of a series of concentrations of Huajiao amide solution $(\mu \mathrm{g} / \mathrm{mL}), 0,50,100,150,200,250,300 \mu \mathrm{g} / \mathrm{mL}$, respectively. Take $20 \mu \mathrm{l}$ to analysis. Standard curve can be made out by using the peak of retention time in the 16-18 min as target peak, with the total peak area as longitudinal ride and correspond of the standard sample concentrations as abscissa (Fig. 5). Curve regression equation was $\mathrm{y}=0.3389 \mathrm{x}$ $\left(\mathrm{R}^{2}=0.9999\right)$.

The technical index of high performance liquid chromatographic (HPLC) method for the detection about detection limit, accuracy degree and precision. The standard curve of nearby limit was $y=0.2586 x+0.0085\left(R^{2}=0.9705\right)$ and the standard deviation was 0.0066 . By the following formula can calculate the limit of detection (DL), it was $0.044 \mu \mathrm{g} / \mathrm{mL}$.

$$
\mathrm{DL}=\frac{3 \sigma-\text { intercept }}{\text { slope }}
$$




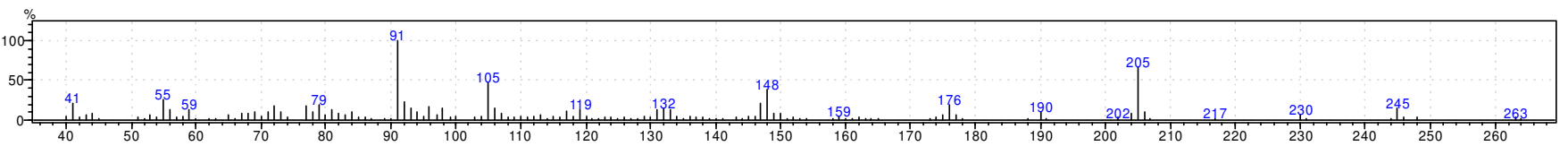

Fig. 5. Mass spectra graph of numb-taste component of Huajiao

$\mathrm{DL}=$ limit of detection, $\sigma=$ standard deviation of blank sample, intercept $=$ the standard curve of nearby limit, slope $=$ the standard curve of nearby limit.

Recovery was determined by adding pepper hemp element standard solution ( $1 \mathrm{~mL}$ ) to the sample fluid (4 mL). Use the following formula to calculate the recovery rate, the result is given in Table-1.

$$
\mathrm{RY}=\frac{\mathrm{SVS}-\mathrm{SV}}{\mathrm{ST}} \times 100 \%
$$

$\mathrm{RY}=$ recovery rate, $\mathrm{SVS}=$ sample values with standard substance, $\mathrm{SV}=$ sample values, $\mathrm{ST}=$ amount of standard substance.

Using the same method multiple times $(n=10)$ determination of certain concentration of the sample solution and calculating the standard deviation and relative standard deviation (C.V.) can determine the precision (Table-2).

$$
\sigma=\sqrt{\frac{\sum_{\mathrm{i}=1}^{\mathrm{n}}\left(\mathrm{x}_{\mathrm{i}}-\overline{\mathrm{x}}\right)^{2}}{\mathrm{n}}} \mathrm{V}_{\sigma}=\frac{\sigma}{\overline{\mathrm{x}}} \times 100 \%
$$

$\sigma=$ Standard deviation of blank sample, $\mathrm{n}=$ the number of observations, $\mathrm{xi}=$ the $\mathrm{i}$ time measured value, $\overline{\mathrm{x}}=$ determination of $\mathrm{n}$ times the average, $\mathrm{V}_{\sigma}=$ relative standard deviation.

Through the above data can be seen the linear correlation of the standard curve is very good, the average recovery rate reached $95 \%$, the relative standard deviation (coefficient of variation) were all less than $5 \%$, its range was $0.79-2.39 \%$. Experi-

\begin{tabular}{|c|c|c|c|c|c|}
\hline \multicolumn{6}{|c|}{$\begin{array}{c}\text { TABLE-1 } \\
\text { RESULTS OF RECOVERY RATE OF NUMB-TASTE MATERIALS BY HPLC }\end{array}$} \\
\hline Number & $\begin{array}{l}\text { Adding quantity } \\
(\mu \mathrm{g} / \mathrm{mL})\end{array}$ & $\begin{array}{c}\text { Content of numb-taste } \\
\text { materials in } \mathrm{A}\end{array}$ & $\begin{array}{l}\text { Content of numb-taste } \\
\text { materials in B }\end{array}$ & $\begin{array}{l}\text { Recovering quantity } \\
(\mu \mathrm{g} / \mathrm{mL})\end{array}$ & $\begin{array}{c}\text { Recovery } \\
(\%)\end{array}$ \\
\hline \multirow{4}{*}{1} & \multirow{4}{*}{ motis } & 56.21 & 65.02 & 8.81 & 88.1 \\
\hline & & 36.42 & 46.76 & 10.34 & 103.4 \\
\hline & & 82.19 & 91.5 & 9.31 & 93.1 \\
\hline & & 61.28 & 71.52 & 10.24 & 102.4 \\
\hline \multirow{4}{*}{2} & \multirow{4}{*}{20} & 56.21 & 75.13 & 18.92 & 94.6 \\
\hline & & 36.42 & 55.73 & 19.31 & 96.6 \\
\hline & & 82.19 & 103.17 & 20.98 & 104.9 \\
\hline & & 61.28 & 80.33 & 19.05 & 95.3 \\
\hline \multirow{4}{*}{3} & \multirow{4}{*}{30} & 56.21 & 85.56 & 29.35 & 97.8 \\
\hline & & 36.42 & 66.99 & 30.57 & 101.9 \\
\hline & & 82.19 & 113.65 & 31.46 & 104.9 \\
\hline & & 61.28 & 91.09 & 29.81 & 99.4 \\
\hline \multirow{4}{*}{4} & \multirow{4}{*}{40} & 56.21 & 96.56 & 40.35 & 100.9 \\
\hline & & 36.42 & 75.38 & 38.96 & 97.4 \\
\hline & & 82.19 & 121.31 & 39.12 & 97.8 \\
\hline & & 61.28 & 101.64 & 40.36 & 100.9 \\
\hline
\end{tabular}
mental results show that this method has good repeatability.

\begin{tabular}{|c|c|c|c|c|c|c|c|c|c|c|c|c|c|}
\hline \multicolumn{14}{|c|}{$\begin{array}{c}\text { TABLE-2 } \\
\text { RESULTS OF REPEATING EXPERIMENT }\end{array}$} \\
\hline \multicolumn{11}{|c|}{ Results determined $(\mu \mathrm{g} / \mathrm{mL})$} & \multirow{2}{*}{ mean } & \multirow{2}{*}{ S.D. } & \multirow{2}{*}{ C.V. $(\%)$} \\
\hline Simple & & & & 4 & 5 & 6 & 7 & 8 & 9 & 10 & & & \\
\hline 1 & 56.21 & 57.35 & 56.1 & 56.78 & 57.89 & 58.12 & 55.87 & 56.32 & 58.12 & 56.17 & 56.89 & 0.90 & 1.57 \\
\hline 2 & 36.25 & 35.71 & 35.28 & 36.15 & 37.34 & 38.08 & 35.7 & 35.98 & 36.07 & 37.15 & 36.37 & 0.87 & 239 \\
\hline 3 & 82.05 & 81.63 & 8097 & 8156 & 81.27 & 82.07 & 83.15 & 81.04 & 80.45 & 81.36 & 81.56 & 0.75 & 0.91 \\
\hline 4 & 61.23 & 64.55 & 63.04 & 64.25 & 61.24 & 62.14 & 61.89 & 63.05 & 61.19 & 62.55 & 6251 & 1.21 & 1.94 \\
\hline 5 & 31.02 & 32.56 & 32.87 & 31.08 & 30.85 & 31.29 & 3254 & 30.56 & 31.28 & 32.89 & 31.69 & 0.91 & 2.87 \\
\hline 6 & 50.26 & 51.25 & 53.08 & 52.84 & 52.47 & 53.07 & 51.14 & 53.65 & 52.17 & 52.89 & 52.28 & 1.07 & 205 \\
\hline 7 & 91.25 & 92.05 & 9354 & 9156 & 91.89 & 92.45 & 93.14 & 91.58 & 9249 & 91.76 & 92.17 & 0.73 & 0.79 \\
\hline 8 & 102.35 & 104.68 & 104.36 & 103.78 & 102.98 & 103.54 & 103.58 & 102.46 & 103.96 & 101.71 & 103.34 & 0.95 & 0.92 \\
\hline 9 & 13259 & 135.48 & 131.69 & 132.94 & 132.81 & 132.95 & 134.16 & 131.05 & 131.95 & 13258 & 13282 & 1.26 & 0.95 \\
\hline 10 & 95.64 & 96.18 & 94.13 & 93.18 & 9245 & 95.18 & 93.67 & 94.68 & 92.18 & 91.79 & 93.91 & 1.51 & 1.61 \\
\hline
\end{tabular}

TABLE-3

\begin{tabular}{|c|c|c|c|}
\hline Sample & Huajiao & Huajiao seasoning oil & Huajiao essential oil \\
\hline Numb-taste contents $(\mu \mathrm{g} / \mathrm{g})$ & 15.15 & 1.68 & 196.65 \\
\hline
\end{tabular}

NUMB-TASTE CONTENTS IN PRICKLY ASH, Z. schinifilium-OIL AND RESIN 
Application of detection methods: Taking just the right amount of Huajiao peel, Huajiao seasoning oil, Huajiao essential oil and samples pretreatment, respectively. With the establishment of HPLC method to the detecting the Huajiao numb-taste component content, the results are given in Table-3.

\section{Conclusion}

Through a new chromatographic technique, the numbtaste component of Huajiao was isolated. At the same time, used GC-MS method to qualitative detection of the separated material, this substance was determined just as numb-taste components. Using this material as a standard reference material, established a HPLC detection method of numb-taste components of Huajiao. Through experimental analysis found that this method was better precision. The establishment of detection method provided excellent technical for identifying the quality of Huajiao.

\section{ACKNOWLEDGEMENTS}

This research was financially supported by the National Natural Science Foundation of China (31391834) and Major Natural Science Foundation of Chongqing (CSTC2006BA1007) and Science and Technology Research Projects of Chongqing Education Commission (KJ31119)

\section{REFERENCES}

1. 1st. and 2 st. Zhong yao zhi, People's Medical Publishing House, Beijing, vol. 3, p. 200 and 365 (1984).

2. K. Mizutani, Y. Fukunaga, O. Tanaka, N. Takasugi, Y. Saruwatari, T. Fuwa, T. Yamauchi, J. Wang, M. Jia, F. Li and Y. Ling, Chem. Pharm. Bull. (Tokyo), 36, 2362 (1988).

3. T. Hatano, K. Inada, T. Ogawa, H. Ito and T. Yoshida, Phytochemistry, 65, 2599 (2004).

4. I. Yasuda, K. Takeya and H. Itokawa, Phytochemistry, 21, 1295 (1982).

5. Q. Xiong, S. Dawen, H. Yamamoto and M. Mizuno, Phytochemistry, 46, 1123 (1997).

6. I.-S. Chen, T.-L. Chen, W.-Y. Lin, I.-L. Tsai and Y.-C. Chen, Phytochemistry, 52, 357 (1999).

7. Y. Kashiwada, C. Ito, H. Katagiri, I. Mase, K. Komatsu, T. Namba and Y. Ikeshiro, Phytochemistry, 44, 1125 (1997).

8. I. Yasuda, K. Takeya and H. Itokawa, Chem. Pharm. Bull. (Tokyo), 29, 1791 (1981).

9. E. Hisatomi, M. Matsui, K. Kubota and A. Kobayashi, J. Agric. Food Chem., 48, 4924 (2000).

10. E.J. Cho, T. Yokozawa, D.Y. Rhyu, S.C. Kim, N. Shibahara and J.C. Park, Phytomedicine, 10, 544 (2003).

11. J.M. Hur, J.G. Park, K.H. Yang, J.C. Park, J.R. Park, S.S. Chun, J.S. Choi and J.W. Choi, Biosci. Biotechnol. Biochem., 67, 945 (2003).

12. T. Sakai, K. Yoshihara and Y. Hirose, Bull. Chem. Soc. Jpn., 41, 1945 (1968).

13. L. Jiang, H. Kojima, K. Yamada, A. Kobayashi and K. Kubota, J. Agric. Food Chem., 49, 5888 (2001).

14. Y. Tezuka, S. Irikawa, T. Kaneko, A.H. Banskota, T. Nagaoka, Q. Xiong, K. Hase and S. Kadota, J. Ethnopharmacol., 77, 209 (2001). 